\title{
Measurements of the production of $W / Z$ boson in association with (heavy flavour) jets with the ATLAS detector
}

\author{
Camilla Vittori, on behalf of the ATLAS Collaboration * \\ INFN and University of Bologna \\ E-mail: camilla.vittoriecern.ch
}

\begin{abstract}
The production of jets in association with a $\mathrm{W}$ or a $\mathrm{Z}$ boson in proton-proton collision is an important process to study QCD in multi-scale environments. Measurements of W/Z boson production in association with heavy flavour quarks provide experimental constraints to improve the theoretical description of these processes, which suffer from larger uncertainties than in the light-jet case. A detailed knowledge of the production of jets associated with electroweak bosons is a key element in the understanding of the Higgs processes, since they represent one of the largest backgrounds for these measurements. Results on the differential production cross sections for W/Z+jets in several kinematic physics observables measured by the ATLAS experiment at the centre of mass energy of $13 \mathrm{TeV}$ are presented and compared to high order QCD calculations and recent Monte Carlo simulations.
\end{abstract}

Sixth Annual Conference on Large Hadron Collider Physics (LHCP2018)

4-9 June 2018

Bologna, Italy

\footnotetext{
${ }^{*}$ Speaker.
} 


\section{Measurement of $\mathbf{W}^{+}$and $W^{-}$cross sections and ratios at $\sqrt{s}=8 \mathrm{TeV}$ with ATLAS}

The $\mathrm{W}(\rightarrow e v)+$ jets production cross section and $\mathrm{W}^{+} / W^{-}$cross section ratios are measured with data collected by ATLAS at $\sqrt{s}=8 \mathrm{TeV}$, corresponding to a luminosity of $20.2 \mathrm{fb}^{-1}$ [1]. Events are selected by requiring one electron with $\mathrm{p}_{T}>20 \mathrm{GeV}$ and $|\eta|<2.47$ (excluding $1.37<$ $|\eta|<1.52)$ and missing transverse energy $\left(\mathrm{E}_{T}^{\text {miss }}\right)$ and transverse mass $\left(\mathrm{m}_{T}\right)$ consistent with the decay of a $\mathrm{W}$ boson $\left(\mathrm{E}_{T}^{\text {miss }}>25 \mathrm{GeV}, \mathrm{m}_{T}>40 \mathrm{GeV}\right)$. Events are required to contain at least one jet with $\mathrm{p}_{T}>30 \mathrm{GeV}$ and rapidity $|y|<4.4$.

The background processes are simulated with Monte Carlo generators [1]. For $\mathrm{W}(\rightarrow e v)+\geq 4$ jets events, the dominant background contribution is multijet (8-16\%), characterised by light jet decaying in electrons and b- or c-hadron decays, with electrons in the final state. This background is estimated through a data-driven technique in a region enriched by multijet events and a fit on data of the $\mathrm{E}_{T}^{\text {miss }}$ distribution is used to extract the multijet template. For events with $\mathrm{W}(\rightarrow e v)+\geq 5-6$ jets, the $t \bar{t}$ background dominates (27-36\%).

The background subtracted data are corrected for detector efficiencies, resolution effects and bin-by-bin migrations to the fiducial phase space by an iterative Bayesian unfolding method. The main sources of systematic uncertainty in the cross section measurements are the Jet Energy Scale (JES) and Resolution (JER). The JES impact ranges from $8 \%$ to $55 \%$ for $\mathrm{N}_{\text {jets }} \geq 1$ to $\geq 7$ and decreases for the $\mathrm{W}^{+}$and $W^{-}$cross section ratio (1\%-17\%). The JER contribution varies from $9 \%$ to $20 \%$ for $\mathrm{N}_{\text {jets }} \geq 1$ to 6 and decreases to $1-5 \%$ for the $\mathrm{W}^{+} / W^{-}$cross section ratios. The dominant uncertainty in the $\mathrm{W}^{+} / W^{-}$cross section ratio measurements is the multijet background modelling ( $1 \%$ to $27 \%$ for $\mathrm{N}_{\text {jets }} \geq 1$ to 6 ), due to statistical uncertainties from the fit on data.
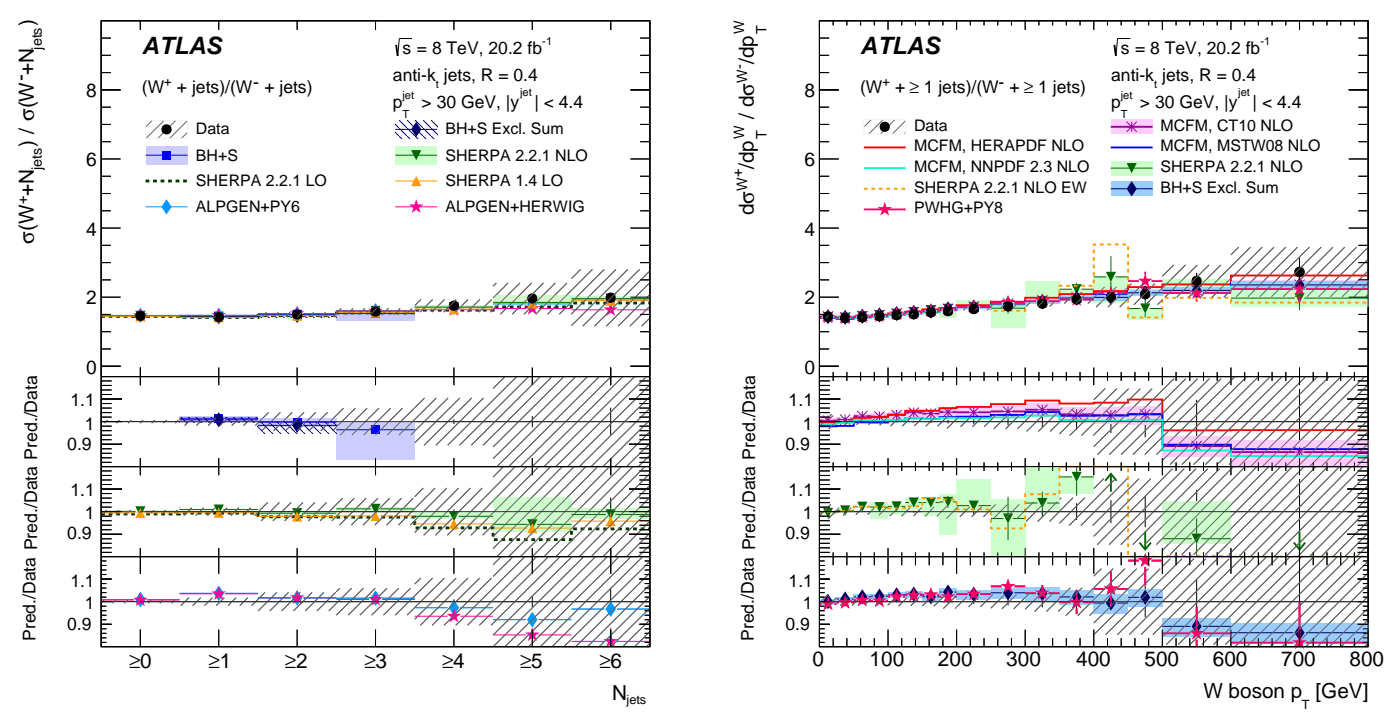

Figure 1: Differential $\mathrm{W}^{+} / W^{-}$cross section ratios as a funtion of the inclusive jet multiplicity (left) and the $\mathrm{W}$ boson $p_{T}$ (right) for the $\mathrm{W}(\rightarrow e v)+$ jets events [1].

The measurements of the cross section ratios for different inclusive jet multiplicities are shown in Fig.1 (left), where an overall data agreement with the predictions, within the uncertainties, can 
be observed. The ALPGEN generator [2] is interfaced with two different parton shower models (HERWIG [3] and Pythia6 [4]). Both predictions show an offset for events with one jet, indicating a problem in the matrix element calculation or in the LO PDF.

Figure 1 (right) shows the cross section ratio distributions as a function of the $\mathrm{W}$ boson $p_{T}$ for $\mathrm{N}_{\text {jets }} \geq 1$. Data are not well described by the NLO SHERPA [5] and the NLO BLACKHAT+SHERPA [6] predictions. The NLO MCFM prediction [7] is shown with four different PDF sets CT10 [8], HERAPDF [9], MSTW08 [10] and NNPDF2.3 [11], whose variations are two times larger with respect to data for $200<\mathrm{p}_{T}^{W}<400 \mathrm{GeV}$. These results would be effective in global PDF fits.

\section{Measurements of the production cross section of a $\mathrm{Z}$ boson in association with jest at $\sqrt{s}=13 \mathrm{TeV}$ with ATLAS}

The measurements of the $\mathrm{Z}+$ jets production cross section are performed with data collected by ATLAS at $\sqrt{s}=13 \mathrm{TeV}$, corresponding to a luminosity of $3.16 \mathrm{fb}^{-1}$ [12]. Events are selected by requiring two opposite charged electrons or muons, passing specific criteria, such as $p_{T}>25 \mathrm{GeV}$ and $|\eta|<2.47$ ( $|\eta|<2.4$ for muons). Jets are reconstructed using the anti-kT algorithm with radius parameter 0.4 and selected with $p_{T}>30 \mathrm{GeV}$ and $|y|<2.5$.

Background contributions from $\mathrm{W}+\mathrm{jets}$, diboson and top processes are estimated using Monte Carlo samples. The multijet background, instead, is estimated through a data driven technique in enriched regions, constructed by loosening the lepton identification and isolation, with a fit on the invariant mass distribution. The dominant background component comes from $t \bar{t}$ production, whose contribution increases from $\sim 1$ to $12 \%$ at high jet multiplicities. The overall purity of the selection ranges from $99 \%$ in the $\mathrm{Z}+\geq 1$ jet sample to $80-85 \%$ in the $\geq 7$ jets.

Cross section measurements are performed in the fiducial region defined by the acceptance of the ATLAS detector. An iterative Bayesian procedure is used to unfold the cross section measurement at particle level, in order to correct for detector inefficiencies and resolutions. Systematics on the unfolding $(\sim 3 \%)$ take into account the limited statistics, the hadronization modelling, the migrations into other kinematic distributions and the finite bin width. Propagated through the unfolding via the migration matrices, the largest contribution to the reconstruction uncertainties is given by jet energy scale ( $8 \%$ in $\mathrm{Z}+\geq 1$ jet up to $25 \%$ in $\mathrm{Z}+\geq 7$ jets).

The electron-muon combined cross section is compared to different predictions. The distribution of the leading-jet $\mathrm{p}_{T}$ in inclusive $\mathrm{Z}+\geq 1,2,3,4$ jet events is shown in Fig.2 (left). While the LO generator MG5_aMC+PY8 CKKWL $[13,14,15]$ models a too hard $\mathrm{p}_{T}$ spectrum, NLO predictions from BLACKHAT+SHERPA, SHERPA and MG5_aMC+Py8 FXFX [16] are in agreement with data over the full range. ALPGEN+PY6 also shows a good agreement with the measured cross section. The $\mathrm{Z}+\geq 1$ jet NNLO prediction [17] well models the spectrum for $\mathrm{Z}+\geq 1$ jet.

The differential cross section as a function of $\mathrm{H}_{T}$ (scalar sum of the $p_{T}$ of all observed final state objects) for $\mathrm{Z}+\geq 1$ jet events is shown in Fig.2 (right). Predictions from SHERPA, ALPGEN+PY6, MG5_aMC+PY8 FXFX well describe the data, while MG5_aMC+PY8 CKKWL overestimates the contribution at large value of $\mathrm{H}_{T}$. Fixed order NLO predictions from BLACKHAT+SHERPA underestimate the cross section for values of $\mathrm{H}_{T}>300 \mathrm{GeV}$, due to the missing contributions from events with higher parton multiplicities. Agreement is recovered by adding higher order in pQCD, as demonstrated by the good description of $\mathrm{H}_{T}$ by $\mathrm{Z}+\geq 1$ jet Njetti NNLO. 

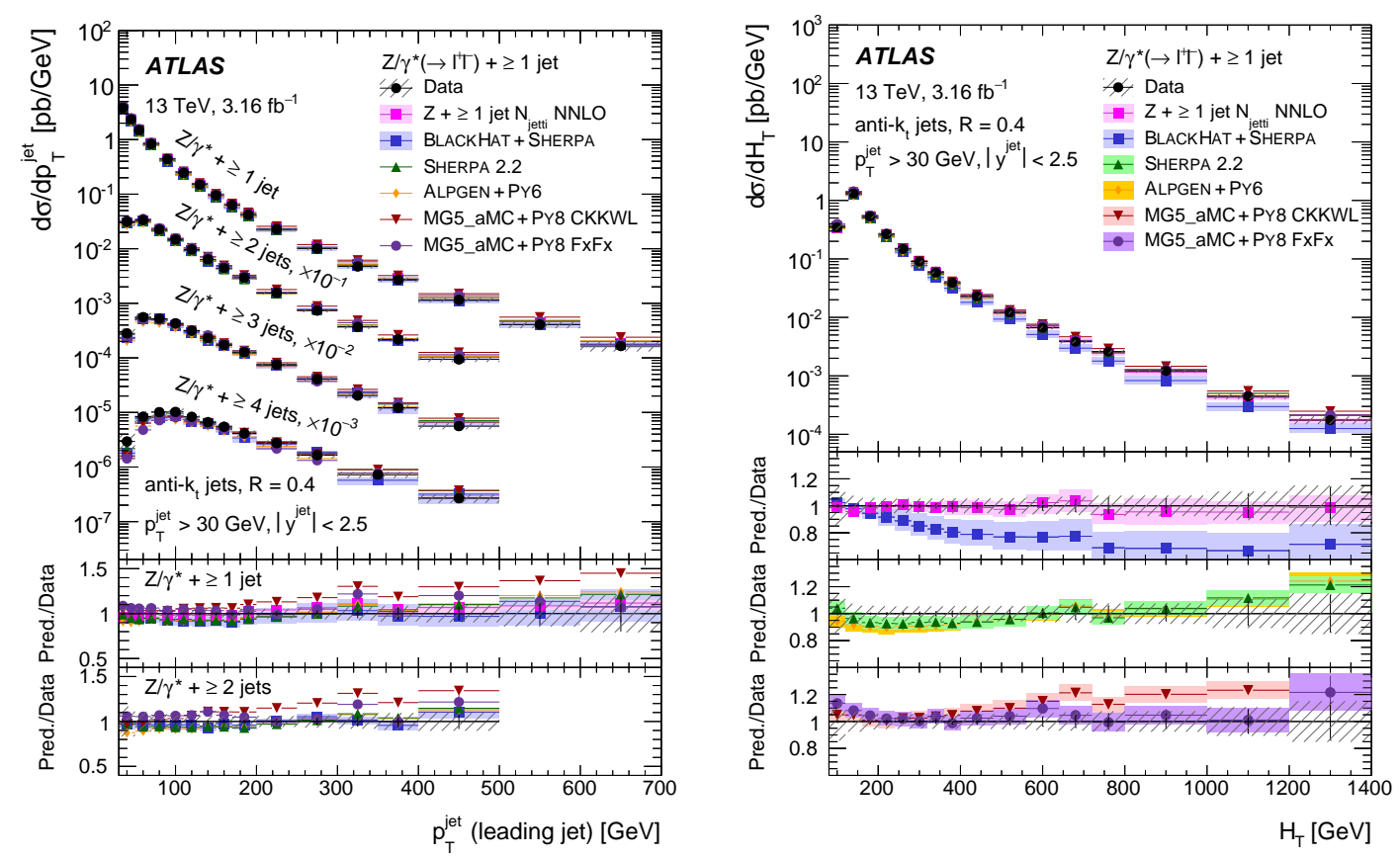

Figure 2: Measured differential cross section as a function of the leading jet $\mathrm{p}_{T}$ (left) and $\mathrm{H}_{T}$ (right) for the Z+jets events [12].

\section{ATLAS simulation of $\mathrm{W} / \mathrm{Z}$ bosons plus jets processes in Run 2}

The ATLAS simulations used to model the production of W/Z boson plus jets in $p p$ collisions are determined at the centre of mass energy $\sqrt{s}=13 \mathrm{TeV}$ [18]. Several predictions are compared, differing in the matrix element, the parton shower model and the scheme used in the HF-jets production. Sherpa 2.2 is a NLO Monte Carlo generator which employs the 5FNS ( 5 Flavour Number Scheme) for the modelling of b-jets. MG5_aMC is a LO generator with the 5FNS for heavy flavour production, interfaced with Pythia8 (MG5_aMC+PY8 CKKWL) for the parton shower. Finally, ALPGEN is a LO prediction with Pythia6 as parton shower model (ALPGEN+Py6) and uses the 4FNS for b-jets prediction.

Simulated $\mathrm{W}+\mathrm{b}$-jets events are selected by requiring one lepton with $p_{T}>25 \mathrm{GeV}$, missing transverse energy $E_{T}^{\text {miss }}>25 \mathrm{GeV}$ and $\mathrm{W}$ transverse mass $m_{T}>60 \mathrm{GeV}$. The differential cross section as a function of the leading b-jet $p_{T}$ for events with $\mathrm{W}+1 \mathrm{~b}$-jet is shown in Fig.3 (left). The cross section from Sherpa 2.2 is systematically higher than that of the two LO-accurate predictions. MG5_aMC+Py8 CKKWL predicts a substantially harder jet- $p_{T}$ spectrum.

Simulated $\mathrm{Z}+\mathrm{b}$-jets events are selected by requiring two opposite charge and same flavour leptons, with $p_{T}>25 \mathrm{GeV},|\eta|<2.5$, whose invariant mass ranges in the window 71-111 GeV. Figure 3 (right) shows the differential cross section distribution as a function of the angular separation of the two b-jets $\left(\Delta R_{b b}\right)$ for Z+2 b-jets events. Sherpa 2.2 and MG5_aMC+Py8 CKKWL provide a similar description of the process within uncertainties, while the 4FNS ALPGEN prediction shows a different shape at low $\Delta R_{b b}$, where the gluon splitting to $b \bar{b}$ pair dominates. Differences among 
the 4FNS and 5FNS Monte Carlo predictions are also observed in the results obtained at the centre of mass energy $\sqrt{s}=7 \mathrm{TeV}$ [19], where none of the theoretical predictions well describe data in the low- $\Delta R_{b b}$ range.
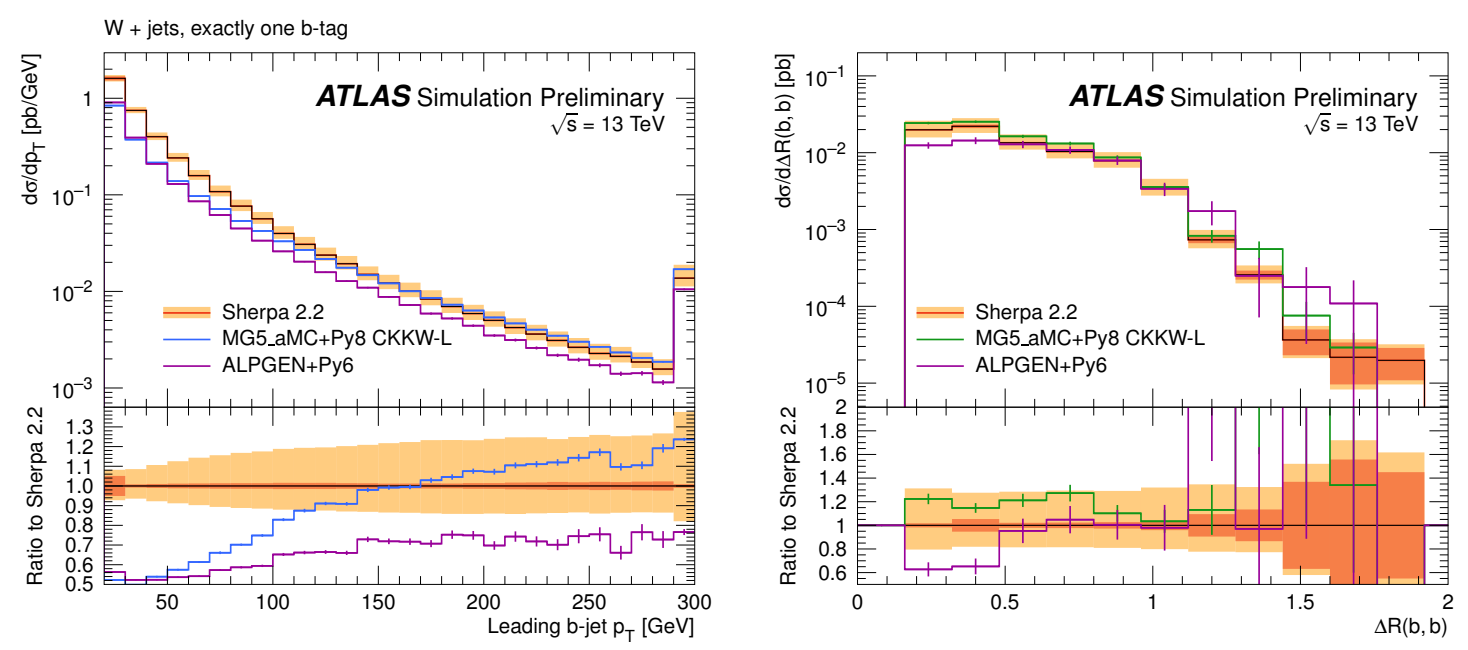

Figure 3: Predictions for differential cross section as a function of leading b-jet $p_{T}$ for $\mathrm{W}+1 \mathrm{~b}$-jet (left) and of the angular separation of the two b-jets $\Delta R_{b b}$ for $\mathrm{Z}+2 \mathrm{~b}$-jets (right) at $\sqrt{s}=13 \mathrm{TeV}$ [18].

\section{Conclusions}

Measurements of $\mathrm{W} / \mathrm{Z}+$ jets in comparison with predictions allow a deep study of the partonic dynamic in the hard scattering and can constrain PDF sets in global fits. When considering bjets, the differences between the 4FNS and 5FNS modes in the prediction of W/Z+b-jets become sensitive to the proton PDF. Therefore, a complementary study on the W/Z+b-jets production at $\sqrt{s}=13 \mathrm{TeV}$ is on-going, with the aim of investigate the proton structure, by comparing predictions based on either 4FNS or 5FNS to the measurements.

\section{References}

[1] ATLAS Collaboration, "Measurement of differential cross sections and $W^{+} W^{-}$cross section ratios for $W$ boson production in association with jets at $\sqrt{s}=8 \mathrm{TeV}$ with the ATLAS detector”, JHEP 05 (2018) 077.

[2] M. L. Mangano, M. Moretti, F. Piccinini, R. Pittau and A. D. Polosa, “ALPGEN, a generator for hard multiparton processes in hadronic collisions”, JHEP 07 (2003) 001.

[3] G. Corcella et al., "HERWIG 6: An Event generator for hadron emission reactions with interfering gluons (including supersymmetric processes)”, JHEP 01 (2001) 010.

[4] T. SjÃüstrand, S. Mrenna and P. Z. Skands, “PYTHIA 6.4 physics and manual”, JHEP 05 (2006) 026.

[5] S. Hoeche, F. Krauss, M. Schonherr and F. Siegert, "QCD matrix elements+parton showers: The NLO case”, JHEP 04 (2013) 027. 
[6] Z. Bern et al., “Next-to-leading order W+5 jet production at the LHC”, Phys. Rev. D 88 (2013) 014025 .

[7] J. M. Campbell, R. K. Ellis and D. L. Rainwater, "Next-to-leading order QCD predictions for W+2 jet and Z+2 jet production at the CERN LHC”, Phys. Rev. D 68 (2003) 094021.

[8] H.-L. Lai et al., “New parton distributions for collider physics”, Phys. Rev. D 82 (2010) 074024.

[9] H1 and ZEUS Collaborations, "Combination of measurements of inclusive deep inelastic ep scattering cross sections and QCD analysis of HERA data”, Eur. Phys. J. C 75 (2015) 580.

[10] A. D. Martin, W. J. Stirling, R. S. Thorne and G. Watt, “Parton distributions for the LHC”, Eur. Phys. J. C 63 (2009) 189.

[11] R. D. Ball et al., “Parton distributions with LHC data”, Nucl. Phys. B 867 (2013) 244.

[12] ATLAS Collaboration, "Measurements of the production cross section of a Z boson in association with jets in pp collisions at $\sqrt{s}=13$ TeV with the ATLAS detector”, Eur. Phys. J. C 77 (2017) 361.

[13] J. Alwall et al., "The automated computation of tree-level and next-to-leading order differential cross sections, and their matching to parton shower simulations”, JHEP 07 (2014) 079.

[14] T. Sjöstrand, S. Mrenna and P. Z. Skands, “A Brief Introduction to PYTHIA 8.1”, Comput. Phys. Commun. 178 (2008) 852.

[15] L. Lonnblad, “Correcting the color dipole cascade model with fixed order matrix elements”, JHEP 05 (2002) 046.

[16] R. Frederix and S. Frixione, “Merging meets matching in MC@NLO”, JHEP 12 (2012) 061.

[17] R. Boughezal et al., "Z-boson production in association with a jet at next-to-next-to-leading order in perturbative QCD”, Phys. Rev. Lett. 116 (2016) 152001.

[18] ATLAS Collaboration, “ATLAS simulation of boson plus jets processes in Run 2", ATL-PHYS-PUB-2017-006.

[19] ATLAS Collaboration, "Measurement of differential production cross-sections for a Z boson in association with b-jets in $7 \mathrm{TeV}$ proton-proton collisions with the ATLAS detector”, HEP 10(2014)141. 